\title{
DO CONSUMO COLABORATIVO A ETNOBOTÂNICA: UMA VISÃO ANTROPOLÓGICA E AMBIENTAL
}

\author{
Ana Cristina Alves Rodrigues ${ }^{1}$ \\ Ivanilza Moreira de Andrade ${ }^{2}$ \\ José Machado Moita Neto ${ }^{3}$
}

Resumo: Temáticas aparentemente distantes podem ser conectadas dentro de uma rede sistêmica de interação. Objetivou-se apresentar uma visão sistêmica que conecta o consumo colaborativo à temática etnobotânica. A metodologia consistiu na análise detalhada do artigo de Lang e Armstrong (2018) e buscas por literaturas em bases de dados. Um mapa conceitual foi produzido e questionamentos foram levantados. Os resultados mostraram que o artigo base interliga as temáticas de etnobotânica, meio ambiente e antropologia, refletindo os valores culturais, modo de vida e comportamento adotado pelas pessoas. Ao optarem pelo consumo colaborativo, sustentável e consciente contribuem diretamente para reduzir os impactos gerados.

Palavras-chave: Meio Ambiente; Pensamento Sistêmico; Rede Sistêmica de Interação.

Abstract: Seemingly distant themes can be connected Environmental Education within a systemic network of interaction. The objective was to present a systemic view that connects collaborative consumption to the ethnobotanical theme. The methodology consisted of a detailed analysis the article by Lang and Armstrong (2018) and searches for literature in databases. A concept map was produced and questions were raised. The results showed that the base article interconnects the themes of ethnobotany, environment and anthropology reflecting cultural values, way of life and behavior adopted by people. When opting for collaborative consumption, sustainable and conscious directly contribute to reducing the impacts generated.

Keywords: Environment; Systemic Thinking; Systemic Interaction Network.

\footnotetext{
1 Universidade Federal do Piauí. E-mail: cristinaalvesrodrigues440@gmail.com. Link para o Lattes: http://lattes.cnpq.br/6920538705073137

2 Universidade Federal Delta do Parnaíba (UFDPar). E-mail: ivanilzaandrade@hotmail.com Link para o Lattes: http://lattes.cnpq.br/7284717480828084

3 Universidade Federal do Piauí E-mail: jose.machado.moita.neto@gmail.com. Link para o Lattes: http://lattes.cnpq.br/5047924139977100
} 


\section{Introdução}

Ao pensar no desenvolvimento sustentável e os seus grandes objetivos para todo o planeta e para as populações mais vulneráveis identificamos a complexidade e magnitude dos Objetivos do Desenvolvimento Sustentável (ODS), os quais, segundo Roma (2019), buscam fazer com que o mundo evolua rapidamente rumo à eliminação de extrema pobreza e em busca da sustentabilidade. Esse debate engloba questões de alcance sistêmico, como financiamento para o desenvolvimento, transferência de tecnologia, capacitação técnica e comércio internacional (PNUD, 2015).

Para o mundo sustentável, conforme Almeida (2002), a atividade econômica, por exemplo, não pode ser pensada e praticada separadamente, e deve estar em permanente diálogo. A sustentabilidade exige uma postura preventiva, pois pode identificar o que um empreendimento pode causar tantos os impactos positivos quanto os negativos. Os avanços tecnológicos que o homem foi capaz de obter tornaram cada vez mais curto o tempo para que os impactos sobre o meio ambiente e sobre a sociedade sejam plenamente sentidos. O autor ainda ressalta que a prática do desenvolvimento sustentável exige uma combinação equilibrada dos mecanismos de comando-e-controle (no que tange às ações e regras governamentais) e autorregulação que significa as iniciativas das empresas para regularem a si mesmas, através do estabelecimento de padrões, monitoramento e metas de redução de impactos ambientais.

Assegurar padrões de produção e consumo sustentáveis, reduzindo substancialmente a geração de resíduos por meio da redução, reciclagem e reuso (PNUD, 2015), traz à tona o consumo colaborativo, que consiste no envolvimento de indivíduos compartilhando seus bens ociosos ou subutilizados com outras pessoas (PISCICELLI; COOPER; FISHER, 2014). Isto significa que mesmo temáticas distantes dentro da seara ambiental como consumo colaborativo e etnobotânica compartilham a mesma rede de conexões. Particularmente, ambas as temáticas dizem respeito ao homem e suas formas de viver e se comunicar com os demais e com o meio que vivem. Todas essas ligações podem ser feitas por meio do pensamento sistêmico.

O conceito do pensamento sistêmico aplica-se ao pensamento voltado para o todo, onde nenhuma ação é empreendida sem se considerar seu impacto em outras áreas da organização e da sociedade. É o quadro de referência conceitual, 0 conjunto de conhecimentos e ferramentas desenvolvidos para esclarecer os padrões como um todo e ajudar a ver como modificá-los efetivamente (CUNHA, 2006). Permite observar todas as relações de interdependências que fazem parte de um processo, o que pode ser utilizado para diagnosticar um problema, surgindo como uma eficiente alternativa de lidar com um cenário de mudanças em uma sociedade globalizada atual (LEITE; LEITE, 2017).

Conforme Ganzer et al. (2014) o pensamento sistêmico integra todas as 
de um todo, não como peças isoladas, bem como criar e mudar a sua realidade. Trata-se de um quadro de referência conceitual onde um conjunto de conhecimentos e ferramentas são desenvolvidos ao longo do tempo.

A compreensão histórica e conceitual acerca do processo de construção do Pensamento Sistêmico, bem como de suas bases estruturantes é imprescindível, pois toda prática está ou deveria estar articulada à compreensão teórica do fenômeno de estudo ou intervenção. Desse modo, ao fazer uso do Pensamento Sistêmico, entende-se que a ciência não pode fornecer uma compreensão completa e definitiva, pois sempre se lida com descrições limitadas e aproximadas da realidade. Tal forma de compreender o mundo e as relações pode ser usada em diversos contextos e em várias áreas do saber (GOMES et al., 2015).

Compreender a realidade considerando vários aspectos permite uma visão ampla e concreta do problema, possibilitando várias vertentes de soluções. Com esta abordagem, pode-se destacar o consumo tradicional de bens e serviços que acontecem dentro de uma ótica individualista e que tem trazido muitos impactos ambientais negativos. O consumo colaborativo surgiu como uma alternativa para mitigar alguns problemas tais como: acúmulo de resíduos, excesso de produção e destruição da matéria-prima. Assim tem transformado progressivamente diversos setores econômicos, incluindo áreas como: a prestação de serviços, aluguel de bens, destinação de usados, compartilhamento de transportes, roupas, dentre outros (VOLGA, 2017).

Diante do exposto, objetivou-se apresentar uma visão sistêmica que liga a temática do consumo colaborativo com a temática da etnobotânica tendo como pano de fundo o desenvolvimento sustentável.

\section{Metodologia}

Para análise detalhada, foi selecionado o texto de Lang e Armstrong (2018), cujo título é "Consumo colaborativo: a influência de liderança de moda, necessidade de exclusividade e materialismo sobre a adoção de aluguel e troca de roupas". Posteriormente, fez-se uma revisão de literatura nas bases de dados: Scielo, ACS Publications, Scopus, Science direct através das palavraschaves: systemic thinking, systemic thinking AND collaborative consumption, systemic thinking AND fashion e ethnobotany AND fashion.

Os artigos foram lidos e destacados os que apresentavam temática mais relevante quanto à abordagem em estudo. Por seguinte, elaborou-se três questionamentos para conduzir ao pensamento sistêmico. artigo?

Questionamento 1- Quais abordagens podem ser relacionadas a este

Questionamento 2- Quais impactos de cunho social e ambiental o consumo colaborativo pode trazer? 
Questionamento 3- Quais relações existem entre o consumo colaborativo abordado no artigo com o estudo Etnobotânico?

Para responder aos questionamentos propostos, foram aprofundadas as temáticas: etnobotânica e consumo colaborativo, bem como a condução ao pensamento sistêmico. Foram realizadas análises e produzido um mapa conceitual (Imagem 1) utilizando as palavras-chaves que interligam consumo colaborativo da moda, etnobotânica e antropologia.

\section{Resultados}

O uso da abordagem sistêmica permite um traçado que interliga e abrange várias vertentes. $\mathrm{O}$ consumo colaborativo está diretamente ligado à sustentabilidade, pois na prática ocorre redução de resíduos, tornando um produto que outrora não era mais "importante" e utilizado por alguém, útil para outras pessoas, com baixo custo que beneficia o meio ambiente e promove consumo mais sustentável.

A etnobotânica, por sua vez, estuda as inter-relações passadas e presentes que se estabelecem entre as pessoas e as plantas (MEDEIROS, 2009). Segundo Queiroz (2014), as fibras naturais, por exemplo, foram utilizadas pelo homem em tempos remotos para a fabricação de fibras e vestimentas desde culturas milenares. Nesse contexto, ao investigar o uso de determinados vegetais por comunidades para fabricação de tecidos, a etnobotânica atua na disseminação de valores culturais e ao mesmo tempo fornecendo informações importantes sobre matéria prima que pode servir para o uso na indústria têxtil. Estes conhecimentos passam a ser aprimorados e utilizados pelas categorias de indústrias de moda na qual sofrem influência das tendências de moda no mercado consumidor.

As grandes lideranças de moda também podem influenciar quanto ao consumo colaborativo, induzindo os consumidores a terem novos comportamentos quanto a compra e uso sustentável de roupas, conforme elencado no mapa conceitual da Figura 1. Assim como os valores culturais, as pessoais também contribuem para determinadas atitudes da sociedade consumidora. Pizzol (2015) apontou uma mistura na adoção ou comportamento associado aos valores pessoais por parte dos consumidores que praticam o consumo colaborativo, uma vez que podem ser observadas médias elevadas em um conjunto de variáveis mais coletivistas como as pertencentes ao universalismo e benevolência, assim como indicadores que evidenciam comportamentos mais individualistas, presentes nas dimensões de auto direcionamento, estimulação e hedonismo. 


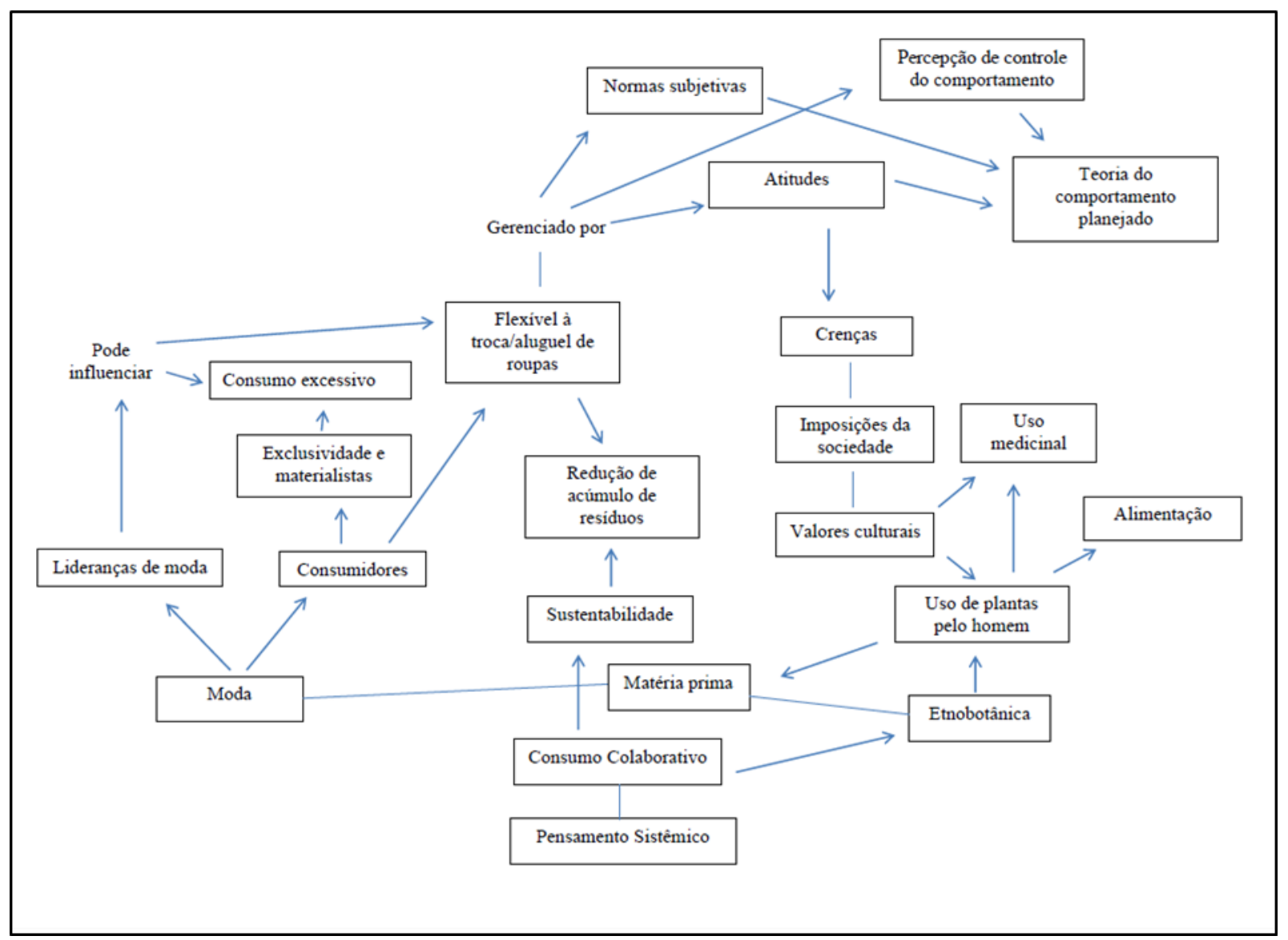

Figura 1: Mapa conceitual sistêmico, abordando as temáticas presentes no artigo de Lang e Armstrong (2018). Fonte: Autores.

Diante dos questionamentos levantados, com base nas análises feitas do artigo de Lang e Armstrong (2018), foram obtidas as seguintes respostas, conforme evidenciados a seguir.

\section{Quais abordagens podem ser relacionadas a este artigo?}

O desenvolvimento das atividades de consumo colaborativo, apesar de participar da atual cultura de mercado, se expressa como uma das tendências que equilibram ou ainda contrariam o consumismo. Muitas abordagens estão relacionadas a este tipo de consumo, podemos destacar o campo de Design como um todo está interessado no processo do Consumo Colaborativo, baseado na comunicação por rede digital, por causa de suas relações com os produtos industrializados reutilizáveis, com a dinâmica social e com o meio ambiente (PAIXÃO; SOUZA, 2015). Podemos considerar os aspectos comportamentais dos entrevistados que se manifestam quanto à decisão de utilizar ou não este tipo de produto. Os aspectos culturais e atitudes também exercem influência mais forte sobre a intenção em relação ao consumo 
colaborativo, o mesmo pode apresentar solução para as preocupações ambientais que envolvem a indústria da moda, promovendo a reutilização de produtos e a redução de novas compras (IRÃ; GEIGER; SCHRADER, 2019; LANG; ARMSTRONG, 2018).

\section{Quais impactos de cunho social e ambiental o consumo colaborativo pode trazer?}

O consumo colaborativo entre diferentes povos pode permitir a interação entre indivíduos que buscam interesses em comum, nutre um senso de comunidade, acolhendo não apenas a diversidade socioeconômica da cidade, mas também cultural, em um esforço, colocando as pessoas em contato umas com as outras e facilitando a vizinhança para gerar condições que contribuam para o conhecimento ecológico com nova aprendizagem, conhecimento e conscientização, melhorando o meio ambiente e tornando o uso mais eficiente dos recursos (WEF, 2017; DUAMANTEA et al., 2019). É um espaço de encontro, diálogo, cumplicidade e confronto, abordando temáticas como economia solidária e agroecologia (PIÑEIRO; SURIÑACH; CASADEVANTE, 2017). Essas formas de consumo demonstram o movimento das pessoas em direção de atividades mais coletivas, abrindo mão da propriedade privada, do individualismo ou do reconhecimento para trabalhar em conjunto, compartilhando experiências, resultados, tempo, dentre outros. Isso demonstra um sentimento de cooperação e a ação coletiva desses consumidores em busca de arranjos não tradicionais (MENEZES, 2015).

As tendências de moda feminina apresentam um curto período de uso e logo são substituídas, gerando acúmulo de resíduos em guarda-roupas e consequentemente serão descartados como lixo. A troca ou aluguel de roupas pode reduzir a quantidade de resíduos gerado pelo consumismo em excesso, além de evitar o descarte inadequado que gera problemas no solo devido ao período entre o descarte e a decomposição, bem como contribuição de entupimento de esgotos e bueiros.

\section{Quais relações existem entre o consumo colaborativo abordado no artigo com o estudo etnobotânico?}

O artigo analisado além de outros aspectos, trás o comportamento humano como um refém de pressões da sociedade. A influência de lideranças de moda pode apresentar um importante fator para desencadear um consumo colaborativo. Além disso, os mecanismos psicológicos dos indivíduos influenciam na participação direta do consumo compartilhado. Segundo Sordi, Petrini e Sampaio (2018), as dimensões mais abstratas podem impactar mais intensamente a atitude do consumidor sobre o consumo compartilhado, enquanto as dimensões mais concretas tendem a influenciar o comportamento com maior intensidade. O consumo sustentável, os riscos e a identidade social, apresentam potencial com níveis mais altos, implicando com maior intensidade as atitudes. 
$\mathrm{Na}$ etnobotânica, essa identidade social é a base para estabelecer as relações existentes entre o homem e os vegetais. É o estudo de populações tradicionais como população urbano-industriais e populações tradicionais como sociedades rurais preocupando-se com as relações entre populações humanas e ambiente botânico (ALBURQUEQUE et al., 2017, p. 11). Nesta abordagem, o homem e a seus aspectos culturais são estudados e relacionados com suas atitudes quanto à utilização, e consumo de vegetais para diversos fins, inclusive para o uso medicinal, reduzindo os custos, tendo em vista que muitas vezes esses vegetais são cultivados em quintais de suas casas. Conforme Colle, Rossato e Zanete (2019), a etnobotânica apresenta papel fundamental para resgatar saberes populares e transmitidos de geração a geração, onde a principal fonte de obtenção de conhecimento dos agentes se originam de pais e avós.

O processo de fabricação de roupas surgiu de povos tradicionais, a utilização dos vegetais para tal era comum, conforme estudo realizado por $\mathrm{Li}$ et al. (2019), os trajes tradicionais e suas técnicas de produção são habilidades amplamente difundidas devido ao vínculo entre têxteis tradicionais e identidade cultural. No entanto, essa prática não pode ser estendida para a produção em massa tendo em vista seu custo temporal para fabricação. Essa prática de extração e tingimento de índigo fazem parte do patrimônio cultural intangível da China.

Muitas espécies são utilizadas por comunidades culturais para diversos fins, seja medicinal, para produção de produtos artesanais, agronegócio, alimentação ou extração de compostos farmacológicos. Conhecer as espécies úteis de uma comunidade, assim como a abundância de indivíduos das espécies é primordial para o planejamento de áreas de conservação de uso sustentável (MAMEDE; PASA, 2019). Dessa forma, questões ambientais como extração e uso de produtos vegetais são equilibradas e menos danosos ao meio ambiente.

O consumo colaborativo também se apresenta como uma grande alternativa para reduzir impactos gerados pelo acúmulo de resíduos, proporcionando interação através de plataformas digitais entre povos e culturas diferentes. A redução de custos quanto a obtenção do produto, a satisfação do cliente ao adquirir um produto bom, de qualidade e por baixo custo impulsionam a busca por esta alternativa.

\section{Conclusões}

O estudo em questão apontou aspectos contidos na temática "Consumo colaborativo: a influência de liderança de moda, necessidade de exclusividade e materialismo sobre a adoção de aluguel e troca de roupas".

Com base nas análises realizadas constatou-se que o consumo colaborativo aplicado à moda se interliga diretamente ao consumo e desenvolvimento sustentável e que em uma visão sistêmica se interliga com a 
etnobotânica e antropologia, refletindo os valores culturais, modo de vida e comportamento adotado pelas pessoas ao optarem ou não pelo consumo colaborativo.

A análise do referido estudo etnobotânico trás à tona como os povos tradicionais utilizam as plantas e sua relação de harmonia com a natureza, mostrando como podemos utilizá-las de forma sustentável, sem deixar de lado o desenvolvimento econômico e a preservação ambiental. Os valores culturais transmitidos de pais para filhos refletem diretamente nos comportamentos de determinadas populações atuais.

Já a utilização de roupas provenientes de aluguel proporciona uma reeducação ambiental quanto à redução de impactos através do consumo. Ações devem ser realizadas para que esta prática seja consolidada não somente por pessoas de alto padrão, mas também por todas as classes sociais. Medidas de exposição e influência através de mídias e de lideranças de moda podem ser um viés para atenuar as agressões ambientais quanto à produção e descarte de roupas.

\section{Agradecimentos}

À Universidade Federal do Piauí, por tornar possível o mestrado do primeiro autor.

\section{Referências}

ALBUQUERQUE, U.P. et al. Ethnobotany for Beginners. Springer International Publishing AG, Buenos Aires, República Argentina, 2017.

ALMEIDA, F. O Bom Negócio da Sustentabilidade. Ed. Nova Fronteira. 2002. Disponível em: <http://www.fernandoalmeida.com.br/livros/livro-fernandoalmeida-sustentabilidade.pdf $>$ Acesso em: 10 out. 2020.

COLLE, M.P.D.; ROSSATO, A.E.; ZANETTE, V.C. Estudo Etnobotânico de Cuphea carthagenensis (Jacq.) J. Macbr. "Sete-Sangrias" (Lythraceae) Junto à Pastoral da Saúde, Santa Catarina. Fronteiras: Journal of Social. v. 8, n. 2. 2019.

CUNHA, A.C.S. Pensamento Sistêmico E Tecnologia Educacional: A Metodologia Webquest. 2006. 131 p. Dissertação. (Mestrado Profissionalizante em Computação) Universidade Estadual do Ceará, Fortaleza, 2006.

DUAMANTE, C.Y. et al. Espacios educativos "otros" y saberes ecológicos: Ferias de intercambio y consumo colaborativo en Chile. Estudios Pedagógicos XLV. № 1: 123-135. 2019.

GANZER, P.P. Aprendizagem de modelo inovador em medicina hospitalar. REN - Revista Escola de Negócios, v.2 n.1, p.48-76. 2014. 
GOMES et al, 2015. As Origens do Pensamento Sistêmico: Das Partes para o Todo. Pensando Famílias. v. 18, n. 2, p. 3-16. Dez, 2014.

IRÃ, S.; GEIGER, S.M.; SCHRADER, U. Consumo colaborativo de moda - um estudo transcultural entre Teerã e Berlim. Journal of Cleaner Production. v. 212, p. 313-3231. 2019.

LANG, C.; ARMSTRONG, C.M.J. Collaborative consumption: The influence of fashion leadership, need for uniqueness, and materialism on female consumers' adoption of clothing renting and swapping. Sustainable Production and Consumption. v. 13 p. 37-47. 2018.

LEITE, D.C.; LEITE, J.F. Diagnóstico De Desafios Ambientais Por Meio De Pensamento Sistêmico Na Escola Estadual Beira Rio, Município De Porto Nacional - To. Revista de Gestão Ambiental e Sustentabilidade - GeAS. vol. 6, n. 3. 2017.

LI, S. et al. Identity blues: the ethnobotany of the indigo dyeing by Landian Yao (lu Mien) in Yunnan, Southwest China. Journal of Ethnobiology and Ethnomedicine. v.15, n. 13. 2019.

MAMEDI, J.S.S.; PASA, M.C. Diversidade e uso de plantas do Cerrado na comunidade São Miguel, Várzea Grande, MT, Brasil. Interações (Campo Grande). v. 20, n. 4. 2019.

MEDEIROS, M.F.T. Etnobotânica Histórica: Pricípios e Procedimentos. Recife. Ed. Nupeea, 2009. 84 p.

MENEZES, U.G. Collaborative Consumption: Relationship Between Trust And Cooperation. Revista Metropolitana de Sustentabilidade - RMS, São Paulo, v. 5, n. 2, p. 95-111. 2015.

PAIXÃO. C.R.; SOUZA, R.P.L. Consumo Colaborativo: Tendência de Construção de Conhecimento e Negócios No Mercado Digital De Produtos Usados. Perspectivas em Gestão \& Conhecimento, João Pessoa, v. 5, n. 2, p. 170-185. 2015.

PIÑEIRO, C.; SURIÑACH, R.; CASADEVANTE, J.L. (Kois) Fernández. Entre el mercado y la cooperación social. Luces y sombras de las prácticas de consumo colaborativo. RES, v. 26, n.1, p. 97-108. 2017.

PISCICELLI, L.; COOPER, T.; FISHER, T.. The role of values in collaborative consumption: insights from a product-service system for lending and borrowing in the UK. Journal of Cleaner Production. 2014. v. 97, 21-29 p. 1-9.

PIZZOL, H.O.D. Proposição de uma escala para mensuração do consumo colaborativo: compreendendo o compartilhamento de bens e a sua relação com os valores pessoais. 2015. 166 p. Dissertação (Mestrado em Administração e Negócios) Faculdade de Administração, Contabilidade e Economia, Universidade Católica do Rio Grande do Sul. Porto Alegre, 2015. 
Programa das Nações Unidas para o Desenvolvimento (PNUD). Acompanhando a agenda 2030 para o desenvolvimento sustentável: subsídios iniciais do Sistema das Nações Unidas no Brasil sobre a identificação de indicadores nacionais referentes aos objetivos de desenvolvimento sustentável/Programa das Nações Unidas para o Desenvolvimento. Brasília: PNUD, 2015. $250 \mathrm{p}$.

QUEIROZ, R.S. Pesquisa e criação: desenvolvimento de coleção de fios de fibras de paina. 2014. 116 p. Dissertação. (Mestrado em Ciências) Universidade de São Paulo. São Paulo, 2014.

ROMA, J.C. Os objetivos de desenvolvimento do milênio e sua transição para os objetivos de desenvolvimento sustentável. Cienc. Cult. v.71, n.1 São Paulo, Jan./Mar. 2019.

SORDI, J.; PETRINI, M.; SAMPAIO, C.H. Concreto ou abstrato? Um esquema conceitual das representações mentais da economia compartilhada. Teoria e Prática em Administração, v. 9, n. 1, p. 105-118. 2018.

VOLGA, A. Influências contraculturais no consumo colaborativo: Insights no contexto do Couchsurfing. Consumer Behavior Review, v. 1, n. 2, 73-81. 2017.

WEF, WORLD ECONOMIC FORUM. Collaboration in Cities: From Sharing to 'Sharing Economy'. 2017. 